\title{
Modelo de optimización para el diseño de una red de distribución del maíz en México
}

\section{Optimization model for the design of a maize distribution network in Mexico}

Miguel Espinoza-Pérez ${ }^{[\mathrm{a}]}$, *Francisca Santana-Robles ${ }^{[\mathrm{b}]}$, Claudia García-Pérez ${ }^{[\mathrm{c}]}$, Rafael GranilloMacías $^{[\mathrm{d}]}$, Isidro Jesús González-Hernández ${ }^{[\mathrm{e}]}$, Arturo Cruz-Avilés ${ }^{[\mathrm{f}]}$, Martín Ortiz-Domínguez ${ }^{[\mathrm{g}]}$

\section{Resumen}

El maíz es uno de los principales alimentos cultivables por sus cualidades alimenticias para consumo humano, agropecuario y uso industrial; por lo que, es representativo en el mercado mundial. México ocupa el séptimo lugar como productor; sin embargo, depende de las importaciones para hacer frente a la demanda nacional. El panorama actual es desafiante para los productores, debido al cambio climático que provoca mayor aleatoriedad con respecto a lluvias y temperatura; así como alta variación en los precios. Esto genera mayor incertidumbre para los productores y reducción en la rentabilidad de sus cultivos. El reto es fortalecer su competitividad a través de diferentes estrategias como reducción de costos de producción y de transporte. El presente trabajo propone un modelo para la optimización de una red de distribución, que selecciona las rutas óptimas tomando en cuenta los costos de transporte y los precios de venta en los mercados demandantes. De acuerdo a los resultados obtenidos, Sinaloa debería surtir a Ciudad de México, Estado de México, Nuevo León, Coahuila, Veracruz, Sonora, San Luis Potosí, Tabasco y Yucatán. Jalisco debería enviar su producción a Ciudad de México, Estado de México, Veracruz y Tabasco; Guerrero debería surtir a Ciudad de México, Veracruz y Tabasco; Chihuahua surtiría a Estado de México y Nuevo León; Guanajuato a Ciudad de México y Estado de México; Tamaulipas surtiría a Nuevo León y Tabasco.

Palabras clave: Optimización, programación lineal, red de distribución, maíz. 


\begin{abstract}
Maize is one of the main crops that can be cultivated due to its nutritional qualities for human, agricultural and industrial use; so, it is representative in the world market. Mexico ranks seventh as a producer; however, it depends on imports to meet domestic demand. The current scenario is challenging for producers, due to climate change that causes greater randomness with respect to rainfall and temperature; as well as high variation in prices. This generates greater uncertainty for the producers and reduction in the profitability of their crops. The challenge is to strengthen its competitiveness through different strategies such as reduction of production and transport costs. The present work proposes a model for the optimization of a distribution network, which selects the optimal routes taking into account transport costs and sales prices in the demanding markets. According to the results obtained, Sinaloa should supply Mexico City, State of Mexico, Nuevo León, Coahuila, Veracruz, Sonora, San Luis Potosí, Tabasco and Yucatán. Jalisco should send its production to Mexico City, State of Mexico, Veracruz and Tabasco; Guerrero should serve Mexico City, Veracruz and Tabasco; Chihuahua would supply the State of Mexico and Nuevo León; Guanajuato to Mexico City and the State of Mexico; Tamaulipas would supply Nuevo León and Tabasco.
\end{abstract}

Key words: Optimization, Linear Programming, Distribution Network, maize.

\title{
Introducción
}

En México, el maíz es el principal alimento para consumo de los habitantes. Según cifras de SIAP (Sistema de Información Agroalimentaria y Pesquera), en el año 2016 se tuvo una producción nacional de 28 millones 250 mil 783.32 toneladas de este grano, siendo los principales estados productores Sinaloa, Jalisco, Michoacán y México.

El productor de maíz se ve afectado en sus ingresos por diferentes causas, siendo ellas el rendimiento de grano y su precio, condiciones climatológicas, bajo acopio del grano en los almacenes que eleva sus costos de transporte, gran cantidad de intermediarios, entre otros factores (Martínez, García \& Mora, 2015). Para un óptimo manejo de inventarios del maíz se debe considerar la logística de abasto, distribución del consumo y producción. Para evitar el almacenamiento debe existir transporte del lugar 
de la producción al mercado y un consumo alto para demandar más producción (Martínez, García \& Mora, 2015).

Las empresas realizan un proceso logístico con el fin de llevar sus productos o servicios desde un origen hasta un destino cubriendo con ciertos requisitos: cantidad, calidad y lugar pactado a un costo mínimo. Las actividades que realizan para cumplir con la demanda de los consumidores son: aprovisionamiento, producción, almacenaje, transporte y distribución, dependiendo si es una empresa industrial, comercial o de servicios (Escudero, 2014).

De acuerdo a la Secretaría de Comunicaciones y Transportes (2013), la infraestructura de transporte en México, está constituida por carretera $(377,659 \mathrm{~km})$, ferrocarriles $(26,727 \mathrm{~km})$ y puertos $(117$ puertos y terminales con 11,122 km de costas), lo que permite la movilización del grano entre diferentes fuentes (zonas productoras) y destinos (centros de acopio y centrales de abasto).

Por otro lado, el lugar donde se deben encontrar los centros de acopio o de distribución, fábricas y almacenes debe ser en sitios establecidos de forma estratégica para reducir distancias y tiempos y de esta forma disminuir todos los costos involucrados de transportar el producto al consumidor final (Ardalan, Karimi, Naderi \& Khamseh, 2016 citados por Paredes, Peláez \& Alarcón, 2016).

En el presente trabajo se propone un modelo de optimización para minimizar los costos de transporte del maíz desde los principales estados productores hasta las centrales de abasto de estados demandantes de este grano en México.

\section{Revisión de la literatura}

Una red de distribución se puede definir como un conjunto de instalaciones (proveedores, plantas y centros de distribución) que intercambian productos e información con el objeto de satisfacer la demanda de los consumidores finales. El diseño de una red es una tarea compleja debido a la falta de metodologías claras para la toma de decisiones a nivel estratégico y táctico.

Para una red logística de distribución se tienen diferentes metodologías para optimizarla y/o diseñarla. Paredes, Peláez \& Alarcón (2016) proponen un modelo de programación lineal entera mixta para diseñar una red óptima de distribución considerando criterios económicos (utilidad anual, costos 
logísticos y gastos como costos de transporte del producto, de la fábrica a un centro de distribución y del centro de distribución al cliente, entre otros), ambientales (emisiones de carbono del vehículo a emplear para transportar el producto y de las instalaciones de producción) y sociales (desempleo). Cabe señalar que a los criterios a considerar se les asigna la misma ponderación.

Reina \& Cortés (2015) proponen una red logística de distribución para el Programa Mercados Campesinos empleando el análisis de decisiones multicriterio que considera criterios financieros (inversión inicial, costo de operación y costo de transporte), de servicio (cumplimiento de pedidos, flexibilidad frente a los cambios y tiempo de respuesta) y efectos (calidad, medio ambiente e identificación de PMC). El uso del análisis de dominancia determina que de las alternativas seleccionadas una no tenga más peso sobre la otra, esto es que una no domine más que otra; también se emplean técnicas de normalización para mantener la proporcionalidad en los criterios.

Soto, Vidal \& Vitor (2014) proponen una metodología combinada para localizar instalaciones. Primero se construye un modelo matemático multicriterio con programación multiobjetivo para generar soluciones factibles. En este modelo matemático se toman en cuenta criterios cuantitativos como costos (de transporte, de distribución, de flota propia o contratada, de inversión, de operación). Después, las soluciones factibles son llevadas a un modelo de decisión multicriterio que considera criterios cualitativos establecidos por los tomadores de decisiones y que finalmente selecciona la mejor alternativa.

Nakandala, Lau \& Zhang (2016) desarrollan un método para minimizar el costo total de transporte de productos alimenticios manteniendo la calidad en niveles aceptables. Los costos que consideran son: costo de transporte, costo de enfriamiento y costo devaluado. El modelo representa desde varias ubicaciones de productores (agricultores) hasta un minorista o almacén en donde se utiliza sólo un vehículo para transportar varios productos con diferentes índices de deterioro de calidad. Toman en cuenta la selección de la ruta, condiciones de enfriamiento en los vehículos y el tiempo de transporte. Utilizan 3 enfoques heurísticos para demostrar la aplicación del modelo de costo total propuesto: Algoritmo Genético (GA), Algoritmo Genético Difuso (FGA) y el Procedimiento Recocido Simulado mejorado (SA).

Por otro lado, de Keizer et al. (2015) proponen un modelo que combina la optimización (MILP Mixed Integer Linear Programming) y la simulación (HOS - Hybrid Optimization-Simulation) para el 
problema del diseño de redes logísticas de flores considerando el deterioro de la calidad del producto desde los productores de flores hasta los minoristas. Entre los productores y minoristas existen centros de distribución llamados hubs que permiten transforman la materia prima (los diferentes tipos de flores) en un producto final (ramos de flores). El deterioro de la calidad está basada en la demanda de los ramos de flores porque puede ser de un tipo de flor o de varios tipos lo que provoca que para su armado dependa de la disponibilidad de cada una de ellas por ser de diferentes proveedores. También tiene que tomarse en cuenta la distancia y los retardos para transportarlas y las variaciones de temperatura. El modelo MILP toma en cuenta la incertidumbre en el suministro, proceso y transporte. Para tomar las decisiones sobre la localización de los hubs y la asignación del flujo del producto, la MILP decide la asignación de las actividades (procesos) en los hubs. La HOS está basada en la simulación de eventos discretos que permite el diseño y configuración de la red de distribución y de igual forma verifica la factibilidad del diseño que arroja MILP.

Drezner \& Scott (2013) proponen un modelo estocástico para minimizar los costos totales que consisten de los costos de transporte y los costos de inventarios que se generan desde la localización de un centro de distribución hasta varios puntos de venta considerando un sólo producto perecedero. El objetivo es minimizar el costo total de tal manera que se encuentre la mejor localización del centro de distribución y la óptima cantidad ordenada económica de producto en cada punto de venta. Los costos de transportar los productos son proporcionales a la distancia entre el centro de distribución y el punto de venta. Se utilizan distancias Euclidianas para localizar los puntos de venta. La demanda en cada punto de venta es continua y su función de densidad es conocida. El modelo de inventario propuesto permite dos ingresos, el precio por producto vendido y un valor de rescate para productos no vendidos. Se aplica el Algoritmo Weiszfeld Generalizado (GWA) para solucionar el problema de las distancias Euclidianas entre el centro de distribución y un punto de venta y la técnica de optimización global Triángulo Pequeño Triángulo Grande (BTST) para obtener una solución al modelo propuesto. Se usan las distribuciones continuas uniforme, exponencial y normal para demostrar la efectividad del algoritmo GWA y de la técnica BTST. 


\section{Materiales y métodos}

Para la optimización de la red de distribución se utilizó programación lineal entera mixta; la programación lineal es una técnica que ayuda a la toma de decisiones a través de modelos matemáticos de optimización; los cuáles son de dos tipos: minimización y maximización. Además, su formulación tiene tres componentes: la función objetivo, restricciones estructurales y restricciones de no negatividad.

Para encontrar la solución óptima del modelo se usó la versión completa de Lingo 9.0 debido a la complejidad del modelo estudiado que involucra una gran cantidad de variables.

\section{Formulación del modelo}

El modelo propuesto tiene como objetivo maximizar las ganancias de los productores de maíz, a través de la asignación óptima de las cantidades a enviar desde cada municipio productor hasta los mercados de los estados demandantes, tomando en cuenta el precio de venta en cada mercado y los costos de transporte. La función objetivo se expresa como

$$
\sum_{i \in I} \sum_{j \in J} x_{i j} p_{j}-\sum_{i \in I} \sum_{j \in J} c_{i j} x_{i j}
$$

En la función objetivo (1), $i \in I$ representa el conjunto de productores de maíz ubicados en los principales estados productores (Sinaloa, Jalisco, Michoacán, Estado de México, Chihuahua, Guanajuato, Guerrero y Tamaulipas). Asimismo, $j \in J$ representa el conjunto de mercados de los estados con mayor de manda de maíz en el país; $x_{i j}$ es la variable de decisión que indica la cantidad de maíz enviada en tonelada del productor $i$ al mercado $j$; por su parte, $p_{j}$ representa el precio de venta en los mercados; $c_{i j}$ indica el costo de transporte (\$/tonelada) desde el productor $i$ al mercado $j$.

Por otro lado, las restricciones de oferta y demanda, están dadas en las ecuaciones (2) y (3), respectivamente; donde $O_{i}$ representa la disponibilidad de maíz para ser enviado, $D_{j}$ indica la demanda en el mercado $\mathrm{j}$. 


$$
\begin{aligned}
& \sum_{i \in I} \sum_{j \in J} x_{i j} \leq O_{i} \\
& \sum_{i \in I} \sum_{j \in J} x_{i j} \geq D_{j}
\end{aligned}
$$

Por último, la restricción de no negatividad de la variable de decisión se representa a través de la ecuación (4), esto garantiza que la variable $x_{i j}$ no tome valores negativos.

$$
x_{i j} \geq 0 \quad \forall i \in I, \forall j \in J
$$

\section{Implementación del modelo}

Los mayores productores de maíz en el año 2016 de acuerdo a (SIAP, 2018) fueron los estados de Sinaloa, Jalisco, México, Guanajuato, Michoacán, Chihuahua, Guerrero y Chiapas. Para esta investigación se calculó el consumo per cápita nacional para poder identificar los estados con mayor desabasto, y aquellos con mayor disponibilidad de maíz después de satisfacer su demanda interna. Para calcular el consumo per cápita se consideró la producción nacional menos las exportaciones más importaciones más autoconsumo, dividido entre la población nacional; resultando un consumo de 236.489 kilogramos por año por persona, para el año 2016. Por lo que, los estados con mayor desabasto resultaron Ciudad de México, México, Nuevo León, Coahuila, Veracruz, Sonora, San Luis Potosí, Tabasco y Yucatán. Asimismo, los estados con mayor oferta son Sinaloa, Jalisco, Michoacán, Chihuahua, Guanajuato, Guerrero y Tamaulipas, véase tablas 1 y 2. 
Tabla 1. Estados con mayor disponibilidad de maíz.

\begin{tabular}{ccc}
\hline Estado & $\begin{array}{c}\text { Municipios } \\
\text { productores }\end{array}$ & $\begin{array}{c}\text { Producción disponible } \\
\text { (ton) }\end{array}$ \\
\hline Sinaloa & 9 & $5,145,019.37$ \\
Jalisco & 9 & $1,231,840.59$ \\
Michoacán & 6 & $434,861.25$ \\
Chihuahua & 6 & $487,000.00$ \\
Guanajuato & 6 & $466,000.00$ \\
Guerrero & 6 & $87,000.00$ \\
Tamaulipas & 6 & $376,100.00$ \\
México & 9 & $845,989.11$
\end{tabular}

Fuente: Elaboración propia

Tabla 2. Estados con mayor desabasto de maíz.

\begin{tabular}{cc}
\hline Estado & $\begin{array}{c}\text { Demanda } \\
\text { (ton) }\end{array}$ \\
\hline Ciudad deMéxico & $2,104,106.82$ \\
México & $1,496,125.14$ \\
Nuevo León & $1,167,301.94$ \\
Coahuila & $663,106.66$ \\
Veracruz & $644,737.50$ \\
Sonora & $502,866.14$ \\
San Luis Potosí & $475,360.46$ \\
Tabasco & $424,101.32$
\end{tabular}

Fuente: Elaboración propia 
Para determinar los precios de venta en cada mercado demandante se consultó la base de datos del Sistema Nacional de Información e Integración de Mercados (SNIIM, 2018) donde observó el comportamiento de los precios del maíz en el periodo de febrero de 2017 a febrero de 2018 en los mercados de los estados demandantes, con esta información se calculó el precio promedio para cada mercado.

Por otro lado, para determinar los costos de envío de los municipios productores a los mercados demandantes, primero se calculó la distancia utilizando la interfaz de programación de aplicaciones Google Matriz Distance, la cual es una herramienta que calcula de manera confiable la distancia entre ubicaciones. Para determinar el costo de transporte se utilizó una tarifa de transporte ferroviario para el maíz, utilizando la siguiente fórmula para el cálculo por tonelada: (Factor variable + Ton-Km) $\mathrm{x}$ $($ Distancia $)+($ Factor Fijo por Ton)

\section{Resultados y discusión}

Para la optimización de la red de transporte se consideró 57 municipios de 8 estados productores de maíz y 12 mercados. De acuerdo a los resultados obtenidos en Lingo 9.0, la solución óptima representa la cantidad de toneladas que debe distribuirse de cada municipio a cada mercado, se maximizan las ganancias de acuerdo a los ingresos menos los costos de transporte.

La tabla 3 muestra los resultados obtenidos del modelo de optimización; donde se observa que Sinaloa ocupa el primer lugar abasteciendo al país, siendo el más autosuficiente y tiene la capacidad de distribuir a 9 estados: Ciudad de México (central de abasto de Iztapalapa), México (central de abasto de Ecatepec y central de abasto de Toluca), Nuevo león (central de abasto de Guadalupe y central de abasto "Estrella" de San Nicolás de los Garza), Coahuila (central de abasto de la Laguna, Torreón), Veracruz (central de abasto de Minatitlán y central de abasto Xalapa), Sonora (central de abasto Francisco I. Madero de Hermosillo), San Luís Potosí (central de abasto de San Luis Potosí), Tabasco (central de abasto de Villahermosa) y Yucatán (central de abasto de Mérida).

Los estados de Jalisco y Michoacán tienen el segundo lugar en importancia abasteciendo a 4 centros de abasto los cuales están ubicados en los estados de Ciudad de México, México, Veracruz y Tabasco. Mientras que, el estado de México debería distribuir a Ciudad de México y Yucatán; el estado de 
Guerrero a Ciudad de México, Veracruz y Tabasco; Chihuahua debería distribuir a los estados de México y Nuevo León; Guanajuato a Ciudad de México y estado de México; finalmente, Tamaulipas enviaría a Nuevo León y Tabasco.

Tabla 3. Distribución óptima del maíz

\begin{tabular}{cc}
\hline Origen & $\begin{array}{c}\text { Destino } \\
\text { (ton) }\end{array}$ \\
\hline Sinaloa & $\begin{array}{c}\text { Ciudad de México, México, Nuevo león, Coahuila, } \\
\text { Veracruz, Sonora, San Luis Potosi, Tabasco, Yucatán }\end{array}$ \\
Jalisco & Ciudad de México, México, Veracruz, Tabasco \\
Michoacán & Ciudad de México, México, Veracruz, Tabasco \\
México & Ciudad de México, México, Yucatán \\
Guerrero & Ciudad de México, Veracruz, Tabasco \\
Chihuahua & Estado de México, Nuevo León \\
Guanajuato & Ciudad de México, México \\
Tamaulipas & Nuevo León, Tabasco \\
\hline
\end{tabular}

Fuente: Elaboración propia

\section{Conclusiones}

El modelo de optimización propuesto para el diseño de una red de distribución permite determinar la forma de distribución óptima del maíz desde los estados ofertantes a los mercados demandantes, de tal manera que, se maximicen las ganancias, considerando el precio de venta y los costos de transporte. Se observó que Ciudad de México es la presenta mayor desabasto; sin embargo, por su ubicación es posible reabastecer la demanda a través de seis estados. Además, Sinaloa es el principal distribuidor a nivel nacional. Debido a los grandes retos a los que se enfrentan los productores, es de suma importancia contar con herramientas que ayuden a una mejor toma de decisiones, lo que representa la contribución de este trabajo. 


\section{Agradecimientos}

Asimismo, los autores le agradecen al Dr. Jorge Zuno Silva, quien es Director de la Escuela Superior de Ciudad Sahagún-UAEH, México, por todas las facilidades para concluir este trabajo de investigación.

\section{Referencias}

De Keizer, Haijema, Bloemhof \& van der Vorst. (2015). Hybrid optimization and simulation to design a logistics network for distributing perishable products. Computers \& Industrial Engineering, $88,26-38$.

Drezner, Z. \& Scott, C. H. (2013). Location of a distribution center for a perishable product. Math Meth Oper Res, 78, 301-314.

Escudero, M. J. (2014). Logística de Almacenamiento. Madrid: Ediciones Paraninfo.

Martínez, A., García, J. A. \& Mora, J. S. (2015). Capacidad de almacenes y demanda de almacenamiento de maíz (Zea mays L.) en el Estado de Chiapas, México. Agrociencia, 49, 689702.

Nakandala, D., Lau, H. \& Zhang, J. (2016). Cost-optimization modelling for fresh food quality and transportation. Industrial Management \& Data Systems, 116(3), 564-583.

Paredes, A. M., Peláez, K. A., \& Alarcón, D. R. (2016). Diseño de una red de distribución de productos perecederos teniendo en cuenta criterios de sostenibilidad. Ingenium, 10(28), 11-17.

Reina, M. L. \& Cortés, F. A. (2015). Selección de una estructura de red logística de distribución para el programa de distribución para el programa mercados campesinos usando técnicas de toma de decisiones multicriterio. Revista Ciencias Estratégicas, 23(33), 89-108.

Secretaría de Comunicaciones y Transportes. (2013). Plan Nacional de Desarrollo 2013-2018 [PDF file]. Recuperado de http://www.sct.gob.mx/fileadmin/banners/Programa_Sectorial_de_Comunicaciones_y_Transpo rtes.pdf

Servicio de Información Agroalimentaria y Pesquera [SIAP] (7 de marzo de 2018). Servicio de Información Agroalimentaria y Pesquera. Disponible en: http://nube.siap.gob.mx/cierre_agricola/

Soto, D., Vidal, J. G. \& Vitor, E. A. (2014). Metodología para localización de centros de distribución a través de análisis multicriterio y optimización. DYNA, 81(184), 28-35. 
[a] Estudiante, Licenciatura en Ingeniería Industrial, Escuela Superior de Ciudad Sahagún, UAEH, Tepeapulco, Hidalgo, México, miguelespinoza534@gmail.com;

${ }^{[b]}$ Licenciatura en Ingeniería Industrial, Escuela Superior de Ciudad Sahagún, UAEH, Tepeapulco, Hidalgo, México, profe_7739@uaeh.edu.mx, 0000-0002-3301-979;

[c] Licenciatura en Ingeniería Industrial, Escuela Superior de Ciudad Sahagún, UAEH, Tepeapulco, Hidalgo, México, cgarciap@ uaeh.edu.mx;

[d] Licenciatura en Ingeniería Industrial, Escuela Superior de Ciudad Sahagún, UAEH, Tepeapulco, Hidalgo, México, rafaelgm@uaeh.edu.mx;

[e] Licenciatura en Ingeniería Industrial, Escuela Superior de Ciudad Sahagún, UAEH, Tepeapulco, Hidalgo, México, igonzalez@uaeh.edu.mx;

[f] Licenciatura en Ingeniería Mecánica, Escuela Superior de Ciudad Sahagún, UAEH, Tepeapulco, Hidalgo, México, arturo_cruz8085@uaeh.edu.mx, 0000-0003-0455-1646;

[g] Licenciatura en Ingeniería Mecánica, Escuela Superior de Ciudad Sahagún, UAEH, Tepeapulco, Hidalgo, México, martin_ortiz@uaeh.edu.mx, 0000-0003-4475-9804. 